\title{
Fixed point theorems for cyclic self-maps involving weaker Meir-Keeler functions in complete metric spaces and applications
}

\author{
Hemant Kumar Nashine ${ }^{1}$ and Salvador Romaguera ${ }^{2 *}$
}

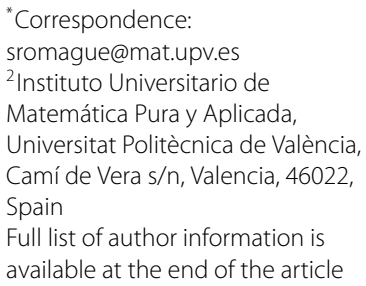

\begin{abstract}
We obtain fixed point theorems for cyclic self-maps on complete metric spaces involving Meir-Keeler and weaker Meir-Keeler functions, respectively. In this way, we extend several well-known fixed point theorems and, in particular, improve some very recent results on weaker Meir-Keeler functions. Fixed point results for well-posed property and for limit shadowing property are also deduced. Finally, an application to the study of existence and uniqueness of solutions for a class of nonlinear integral equations is presented.
\end{abstract}

MSC: $47 \mathrm{H} 10 ; 54 \mathrm{H} 25 ; 54 \mathrm{E} 50 ; 45 \mathrm{G} 10$

Keywords: fixed point; cyclic map; weaker Meir-Keeler function; complete metric space; integral equation

\section{Introduction}

In their paper [1], Kirk, Srinavasan and Veeramani started the fixed point theory for cyclic self-maps on (complete) metric spaces. In particular, they obtained, among others, cyclic versions of the Banach contraction principle [2], of the Boyd and Wong fixed point theorem [3] and of the Caristi fixed point theorem [4]. From then, several authors have contributed to the study of fixed point theorems and best proximity points for cyclic contractions (see, e.g., [5-13]). Very recently, Chen [14] (see also [15]) introduced the notion of a weaker Meir-Keeler function and obtained some fixed point theorems for cyclic contractions involving weaker Meir-Keeler functions.

In this paper we obtain a fixed point theorem for cyclic self-maps on complete metric spaces involving Meir-Keeler functions and deduce a variant of it for weaker Meir-Keeler functions. In this way, we extend in several directions and improve, among others, the main fixed point theorem of Chen's paper [14, Theorem 3]. Some consequences are given after the main results. Fixed point results for well-posedness property and for limit shadowing property in complete metric spaces are also given. Finally, an application to the study of existence and uniqueness of solution for a class of nonlinear integral equations is presented.

We recall that a self-map $f$ of a (non-empty) set $X$ is called a cyclic map if there exists $m \in$ $\mathbb{N}$ such that $X=\bigcup_{i=1}^{m} A_{i}$, with $A_{i}$ non-empty and $f\left(A_{i}\right) \subseteq A_{i+1}, i=1, \ldots, m$, where $A_{m+1}=A_{1}$. In this case, we say that $X=\bigcup_{i=1}^{m} A_{i}$ is a cyclic representation of $X$ with respect to $f$.

( 2013 Nashine and Romaguera; licensee Springer. This is an Open Access article distributed under the terms of the Creative Commons Attribution License (http://creativecommons.org/licenses/by/2.0), which permits unrestricted use, distribution, and reproduction in any medium, provided the original work is properly cited. 


\section{Fixed point results}

In the sequel, the letters $\mathbb{R}, \mathbb{R}^{+}$and $\mathbb{N}$ will denote the set of real numbers, the set of nonnegative real numbers and the set of positive integer numbers, respectively.

Meir and Keeler proved in [16] that if $f$ is a self-map of a complete metric space $(X, d)$ satisfying the condition that for each $\varepsilon>0$ there is $\delta>0$ such that, for any $x, y \in X$, with $\varepsilon \leq d(x, y)<\varepsilon+\delta$, we have $d(f x, f y)<\varepsilon$, then $f$ has a unique fixed point $z \in X$ and $f^{n} x \rightarrow z$ for all $x \in X$.

This important result suggests the notion of a Meir-Keeler function:

A function $\phi: \mathbb{R}^{+} \rightarrow \mathbb{R}^{+}$is said to be a Meir-Keeler function if for each $\varepsilon>0$, there exists $\delta>0$ such that for $t>0$ with $\varepsilon \leq t<\varepsilon+\delta$, we have $\phi(t)<\varepsilon$.

Remark 1 It is obvious that if $\phi$ is a Meir-Keeler function, then $\phi(t)<t$ for all $t>0$.

In [14], Chen introduced the following interesting generalization of the notion of a MeirKeeler function.

Definition 1 [14, Definition 3] A function $\phi: \mathbb{R}^{+} \rightarrow \mathbb{R}^{+}$is called a weaker Meir-Keeler function if for each $\varepsilon>0$, there exists $\delta>0$ such that for $t>0$ with $\varepsilon \leq t<\varepsilon+\delta$, there exists $n_{0} \in \mathbb{N}$ such that $\phi^{n_{0}}(t)<\varepsilon$.

Now let $\phi, \varphi: \mathbb{R}^{+} \rightarrow \mathbb{R}^{+}$. According to Chen [14, Section 2], consider the following conditions for $\phi$ and $\varphi$, respectively.

$\left(\phi_{1}\right) \phi(t)=0 \Leftrightarrow t=0$

$\left(\phi_{2}\right)$ for all $t>0$, the sequence $\left\{\phi^{n}(t)\right\}_{n \in \mathbb{N}}$ is decreasing;

$\left(\phi_{3}\right)$ for $t_{n}>0$,

(a) if $\lim _{n \rightarrow \infty} t_{n}=\gamma>0$, then $\lim _{n \rightarrow \infty} \phi\left(t_{n}\right)<\gamma$, and

(b) if $\lim _{n \rightarrow \infty} t_{n}=0$, then $\lim _{n \rightarrow \infty} \phi\left(t_{n}\right)=0$;

$\left(\varphi_{1}\right) \varphi$ is non-decreasing and continuous with $\varphi(t)=0 \Leftrightarrow t=0$;

$\left(\varphi_{2}\right) \varphi$ is subadditive, that is, for every $t_{1}, t_{2} \in \mathbb{R}^{+}, \varphi\left(t_{1}+t_{2}\right) \leq \varphi\left(t_{1}\right)+\varphi\left(t_{2}\right)$;

$\left(\varphi_{3}\right)$ for $t_{n}>0, \lim _{n \rightarrow \infty} t_{n}=0$ if and only if $\lim _{n \rightarrow \infty} \varphi\left(t_{n}\right)=0$.

Definition 2 [14, Definition 4] Let $(X, d)$ be a metric space. A self-map $f$ of $X$ is called a cyclic weaker $(\phi \circ \varphi)$-contraction if there exist $m \in \mathbb{N}$, for which $X=\bigcup_{i=1}^{m} A_{i}$ (each $A_{i}$ a nonempty closed set), and two functions $\phi, \varphi: \mathbb{R}^{+} \rightarrow \mathbb{R}^{+}$satisfying conditions $\left(\phi_{i}\right), i=1,2,3$, and $\left(\varphi_{i}\right), i=1,2,3$, respectively, with $\phi$ a weaker Meir-Keeler function such that

(1) $X=\bigcup_{i=1}^{m} A_{i}$ is a cyclic representation of $X$ with respect to $f$;

(2) for any $x \in A_{i}, y \in A_{i+1}, i=1,2, \ldots, m$,

$$
\varphi(d(f x, f y)) \leq \phi(\varphi(d(x, y)))
$$

where $A_{m+1}=A_{1}$.

By using the above concept, Chen established the following fixed point theorem.

Theorem 1 [14, Theorem 3] Let $(X, d)$ be a complete metric space. Then every cyclic weaker $(\phi \circ \varphi)$-contraction $f$ of $X$ has a unique fixed point $z$. Moreover, $z \in \bigcap_{i=1}^{m} A_{i}$, where $X=$ $\bigcup_{i=1}^{m} A_{i}$ is the cyclic representation of $X$ with respect to $f$ of Definition 2. 
We shall establish fixed point theorems which improve in several directions the preceding theorem. To this end, we start by obtaining a fixed point theorem for cyclic contractions involving Meir-Keeler functions.

Theorem 2 Let $f$ be a self-map of a complete metric space $(X, d)$, and let $X=\bigcup_{i=1}^{m} A_{i}$ be a cyclic representation of $X$ with respect to $f$, with $A_{i}$ non-empty and closed, $i=1, \ldots, m$. If $\phi: \mathbb{R}^{+} \rightarrow \mathbb{R}^{+}$is a Meir-Keeler function such that for any $x \in A_{i}, y \in A_{i+1}, i=1,2, \ldots, m$,

$$
d(f x, f y) \leq \phi(d(x, y))
$$

where $A_{m+1}=A_{1}$, then f has a unique fixed point $z$. Moreover, $z \in \bigcap_{i=1}^{m} A_{i}$.

Proof Let $x_{0} \in A_{m}$. For each $n \in \mathbb{N} \cup\{0\}$, put $x_{n}=f^{n} x_{0}$. Note that $x_{n m+i} \in A_{i}$ whenever $n \in \mathbb{N} \cup\{0\}$ and $i=1,2, \ldots, m$.

If $x_{n_{0}}=x_{n_{0}+1}$ for some $n_{0}$, then $x_{n}$ is a fixed point of $f$. So, we assume that $x_{n} \neq x_{n+1}$ for all $n \in \mathbb{N} \cup\{0\}$. By Remark 1 and the contraction condition, it follows that $\left\{d\left(x_{n}, x_{n+1}\right)\right\}_{n \in \mathbb{N}}$ is a strictly decreasing sequence in $\mathbb{R}^{+}$, so there exists $r \in \mathbb{R}^{+}$such that $\lim _{n \rightarrow \infty} d\left(x_{n}, x_{n+1}\right)=r$. If $r>0$, there is $n_{0} \in \mathbb{N}$ such that $\phi\left(d\left(x_{n}, x_{n+1}\right)\right)<r$ for all $n \geq n_{0}$ by our assumption that $\phi$ is a Meir-Keeler function. Hence, $d\left(x_{n+1}, x_{n+2}\right)<r$ for all $n \geq n_{0}$, a contradiction. Therefore $\lim _{n \rightarrow \infty} d\left(x_{n}, x_{n+1}\right)=0$.

Next we prove that $\left\{x_{n}\right\}_{n \in \mathbb{N}}$ is a Cauchy sequence in $(X, d)$. Choose an arbitrary $\varepsilon>0$. Then, there is $\delta \in(0, \varepsilon)$ such that for $t>0$ with $\varepsilon \leq t<\varepsilon+\delta$, we have $\phi(t)<\varepsilon$. Let $k_{0} \in \mathbb{N}$ be such that $d\left(x_{k}, x_{k+1}\right)<\delta / 2, d\left(x_{k}, x_{k+m-1}\right)<\varepsilon / 2$ and $d\left(x_{k}, x_{k+m+1}\right)<\delta / 2$ for all $k \geq k_{0}$.

Take any $k>k_{0}$. Then $k=n m+i$ for some $n \in \mathbb{N}$ and some $i \in\{1,2, \ldots, m\}$. By induction we shall show that $d\left(x_{n m+i}, x_{(n+j) m+i+1}\right)<\varepsilon$ for all $j \in \mathbb{N}$.

Indeed, for $j=1$, we have

$$
d\left(x_{n m+i}, x_{n m+i+m+1}\right)=d\left(x_{k}, x_{k+m+1}\right)<\frac{\delta}{2}<\varepsilon .
$$

Now, assume that $d\left(x_{n m+i}, x_{(n+j) m+i+1}\right)<\varepsilon$ for some $j \in \mathbb{N}$. Thus

$$
\begin{aligned}
d\left(x_{n m+i-1}, x_{(n+j+1) m+i}\right) \leq & d\left(x_{n m+i-1}, x_{n m+i}\right)+d\left(x_{n m+i}, x_{(n+j) m+i+1}\right) \\
& +d\left(x_{(n+j) m+i+1}, x_{(n+j+1) m+i}\right) \\
< & \frac{\delta}{2}+\varepsilon+\frac{\delta}{2}=\delta+\varepsilon .
\end{aligned}
$$

If $\varepsilon \leq d\left(x_{n m+i-1}, x_{(n+j+1) m+i}\right)$, then $\phi\left(d\left(x_{n m+i-1}, x_{(n+j+1) m+i}\right)\right)<\varepsilon$, and, by the contraction condition,

$$
d\left(x_{n m+i}, x_{(n+j+1) m+i+1}\right)<\varepsilon .
$$

If $d\left(x_{n m+i-1}, x_{(n+j+1) m+i}\right)<\varepsilon$, we deduce

$$
\begin{aligned}
d\left(x_{n m+i}, x_{(n+j+1) m+i+1}\right) & \leq \phi\left(d\left(x_{n m+i-1}, x_{(n+j+1) m+i}\right)\right) \\
& <d\left(x_{n m+i-1}, x_{(n+j+1) m+i}\right)<\varepsilon .
\end{aligned}
$$


It immediately follows that $\left\{x_{n}\right\}_{n \in \mathbb{N}}$ is a Cauchy sequence in $(X, d)$. Hence, there exists $z \in X$ such that $x_{n} \rightarrow z$. Since each $A_{i}$ is closed, we deduce that $z \in \bigcap_{i=1}^{m} A_{i}$.

Moreover, $z=f z$. Indeed, let $i_{0} \in\{1, \ldots, m\}$ be such that $f z \in A_{i_{0}+1}$. Then

$$
\begin{aligned}
d(z, f z) & \leq d\left(z, x_{n m+i_{0}}\right)+d\left(x_{n m+i_{0}}, f z\right) \leq d\left(z, x_{n m+i_{0}}\right)+\phi\left(d\left(x_{n m+i_{0}-1}, z\right)\right) \\
& <d\left(z, x_{n m+i_{0}}\right)+d\left(x_{n m+i_{0}-1}, z\right)
\end{aligned}
$$

for all $n \in \mathbb{N}$. Since $\lim _{n \rightarrow \infty} d\left(z, x_{n m+i_{0}}\right)=\lim _{n \rightarrow \infty} d\left(z, x_{n m+i_{0}-1}\right)=0$, it follows that $d(z, f z)=$ 0, i.e., $z=f z$.

Finally, let $u \in X$ with $u=f u$ and $u \neq z$. Since $z \in \bigcap_{i=1}^{m} A_{i}$, we have $d(f z, f u) \leq \phi(d(z, u))$, so $d(z, u)<d(z, u)$, a contradiction. Hence $u=z$, and thus $z$ is the unique fixed point of $f$.

Next we analyze some relations between Chen's conditions $\left(\phi_{i}\right), i=1,2,3$.

Lemma 1 If $\phi: \mathbb{R}^{+} \rightarrow \mathbb{R}^{+}$satisfies $\left(\phi_{3}\right)(\mathrm{a})$, then $\phi$ is a Meir-Keeler function that satisfies conditions $\left(\phi_{2}\right)$ and $\left(\phi_{3}\right)(\mathrm{b})$.

Proof Suppose that $\phi$ is not a Meir-Keeler function. Then there exists $\varepsilon>0$ such that for each $n \in \mathbb{N}$ we can find a $t_{n}>0$ with $\varepsilon \leq t_{n}<\varepsilon+1 / n$ and $\phi\left(t_{n}\right) \geq \varepsilon$. Then $\lim _{n \rightarrow \infty} t_{n}=\varepsilon>0$, but $\phi\left(t_{n}\right) \geq \varepsilon$ for all $n$, so condition $\left(\phi_{3}\right)($ a) is not satisfied. We conclude that condition $\left(\phi_{3}\right)$ (a) implies that $\phi$ is a Meir-Keeler function. Hence, by Remark $1, \phi(t)<t$ for all $t>0$, so the sequence $\left\{\phi^{n}(t)\right\}_{n \in \mathbb{N}}$ is (strictly) decreasing for all $t>0$, and thus condition $\left(\phi_{2}\right)$ is satisfied. Finally, if $\lim _{n \rightarrow \infty} t_{n}=0$, with $t_{n}>0$, we deduce that $\lim _{n \rightarrow \infty} \phi\left(t_{n}\right)=0$ because $\phi\left(t_{n}\right)<t_{n}$ for all $n$, so condition $\left(\phi_{3}\right)(\mathrm{b})$ also holds.

Proposition 1 Let $\varphi: \mathbb{R}^{+} \rightarrow \mathbb{R}^{+}$be a function satisfying conditions $\left(\varphi_{1}\right)$ and $\left(\varphi_{2}\right)$. If $(X, d)$ is a metric space, then the function $p: X \times X \rightarrow \mathbb{R}^{+}$, given by

$$
p(x, y)=\varphi(d(x, y))
$$

is a metric on $X$. If, in addition, $(X, d)$ is complete and $\varphi$ satisfies condition $\left(\varphi_{3}\right)$, then the metric space $(X, p)$ is complete.

Proof We first show that $p$ is a metric on $X$. Let $x, y, z \in X$ :

- Suppose $p(x, y)=0$. Then $\varphi(d(x, y))=0$, so $d(x, y)=0$ by $\left(\varphi_{1}\right)$. Hence $x=y$.

- Clearly, $p(x, y)=p(y, x)$.

- Since $d(x, y) \leq d(x, z)+d(z, y)$, and $\varphi$ is non-decreasing and subadditive, we deduce that $\varphi(d(x, y)) \leq \varphi(d(x, z))+\varphi(d(z, y))$, i.e., $p(x, y) \leq p(x, z)+p(z, y)$.

Finally, suppose that $(X, d)$ is complete with $\varphi$ satisfying $\left(\varphi_{i}\right), i=1,2,3$. Let $\left\{x_{n}\right\}_{n \in \mathbb{N}}$ be a Cauchy sequence in $(X, p)$. If $\left\{x_{n}\right\}_{n \in \mathbb{N}}$ is not a Cauchy sequence in $(X, d)$, there exist $\varepsilon>0$ and sequences $\left\{n_{k}\right\}_{k \in \mathbb{N}}$ and $\left\{m_{k}\right\}_{k \in \mathbb{N}}$ in $\mathbb{N}$ such that $k<n_{k}<m_{k}<n_{k+1}$ and $d\left(x_{n_{k}}, x_{m_{k}}\right) \geq$ $\varepsilon$ for all $k \in \mathbb{N}$. By $\left(\varphi_{3}\right)$, the sequence $\left\{p\left(x_{n_{k}}, x_{m_{k}}\right)\right\}_{k \in \mathbb{N}}$ does not converge to zero, which contradicts the fact that $\left\{x_{n}\right\}_{n \in \mathbb{N}}$ is a Cauchy sequence in $(X, p)$. Consequently, $\left\{x_{n}\right\}_{n \in \mathbb{N}}$ is a Cauchy sequence in $(X, d)$, so it converges in $(X, d)$ to some $x \in X$. From $\left(\varphi_{3}\right)$ we deduce that $\left\{x_{n}\right\}_{n \in \mathbb{N}}$ converges to $x$ in $(X, p)$. Therefore $(X, p)$ is a complete metric space. 
Remark 2 Note that the continuity of $\varphi$ is not used in the preceding proposition.

Now we easily deduce the following improvement of Chen's theorem.

Theorem 3 Let $f$ be a self-map of a complete metric space $(X, d)$, and let $X=\bigcup_{i=1}^{m} A_{i}$ be a cyclic representation of $X$ with respect to $f$, with $A_{i}$ non-empty and closed, $i=1, \ldots, m$. If $\phi, \varphi: \mathbb{R}^{+} \rightarrow \mathbb{R}^{+}$satisfy conditions $\left(\phi_{3}\right)(\mathrm{a})$ and $\left(\varphi_{i}\right), i=1,2,3$, respectively, and for any $x \in A_{i}$, $y \in A_{i+1}, i=1,2, \ldots, m$, it follows

$$
\varphi(d(f x, f y)) \leq \phi(\varphi(d(x, y)))
$$

where $A_{m+1}=A_{1}$, then f has a unique fixed point $z$. Moreover, $z \in \bigcap_{i=1}^{m} A_{i}$.

Proof Define $p: X \times X \rightarrow \mathbb{R}^{+}$by $p(x, y)=\varphi(d(x, y))$ for all $x, y \in X$. By Proposition $1,(X, p)$ is a complete metric space. Moreover, from the condition

$$
\varphi(d(f x, f y)) \leq \phi(\varphi(d(x, y)))
$$

for all $x \in A_{i}, y \in A_{i+1}, i=1, \ldots, m$, it follows that

$$
p(f x, f y)=\varphi(d(f x, f y)) \leq \phi(\varphi(d(x, y)))=\phi(p(x, y))
$$

for all $x \in A_{i}, y \in A_{i+1}, i=1, \ldots, m$.

Finally, since by Lemma $1 \phi$ is a Meir-Keeler function, we can apply Theorem 2 , so there exists $z \in \bigcap_{i=1}^{m} A_{i}$, which is the unique fixed point of $f$.

Note that the continuity of $\varphi$ can be omitted in Theorem 3. Moreover, the condition that $\phi$ is a weaker Meir-Keeler function turns out to be irrelevant by virtue of Lemma 1 . This fact suggests the question of obtaining a fixed point theorem for cyclic contractions involving explicitly weaker Meir-Keeler functions. In particular, it is natural to wonder if Theorem 2 remains valid when we replace 'Meir-Keeler function' by 'weaker Meir-Keeler function. In the sequel we answer this question. First we give an easy example which shows that it has a negative answer in general, but the answer is positive whenever the weaker Meir-Keeler function is non-decreasing as Theorem 5 below shows.

Example 1 Let $X=\{0,1\}$ and let $d$ be the discrete metric on $X$, i.e., $d(0,0)=d(1,1)=0$ and $d(x, y)=1$ otherwise. Of course $(X, d)$ is a complete metric space. Define $f: X \rightarrow X$ by $f 0=1$ and $f 1=0$, and consider the function $\phi: \mathbb{R}^{+} \rightarrow \mathbb{R}^{+}$defined by $\phi(t)=t / 2$ for all $t \in$ $[0,1), \phi(1)=2$ and $\phi(t)=1 / 2$ for all $t>1$. Clearly, $\phi$ is a weaker Meir-Keeler function (note, in particular, that $\left.\phi^{2}(1)=1 / 2<1\right)$, but it is not a Meir-Keeler function because $\phi(1)>1$. Finally, since $d(f 0, f 1)=1$ and $\phi(d(0,1))=2$, we deduce that $d(f x, f y) \leq \phi(d(x, y))$ for all $x, y \in X$. However, $f$ has no fixed point.

The function $\phi$ of the preceding example is not non-decreasing. This fact is not casual as Theorem 5 below shows.

Lemma 2 Let $\phi: \mathbb{R}^{+} \rightarrow \mathbb{R}^{+}$be a non-decreasing weaker Meir-Keeler function. Then the following hold: 
(i) $\phi(t)<t$ for all $t>0$;

(ii) $\lim _{n \rightarrow \infty} \phi^{n}(t)=0$ for all $t>0$.

Proof (i) Suppose that there exists $t_{0}>0$ such that $t_{0} \leq \phi\left(t_{0}\right)$. Since $\phi$ is non-decreasing, we deduce that $\left\{\phi^{n}\left(t_{0}\right)\right\}_{n \in \mathbb{N} \cup\{0\}}$ is a non-decreasing sequence in $\mathbb{R}^{+}$, so, in particular, $t_{0} \leq$ $\phi^{n}\left(t_{0}\right)$ for all $n \in \mathbb{N}$. Finally, since $\phi$ is a weaker Meir-Keeler function, there exists $n_{0} \in \mathbb{N}$ such that $\phi^{n_{0}}\left(t_{0}\right)<t_{0}$, which yields a contradiction.

(ii) Fix $t>0$. By (i) the sequence $\left\{\phi^{n}(t)\right\}_{n \in \mathbb{N}}$ is (strictly) decreasing, so there exists $r \geq 0$ such that $r=\lim _{n \rightarrow \infty} \phi^{n}(t)$. If $r>0$, there is $\delta>0$ such that for $s>0$ with $r \leq s<r+\delta$, there exists $n_{s} \in \mathbb{N}$ with $\phi^{n_{s}}(s)<r$. Let $n_{r} \in \mathbb{N}$ be such that $r<\phi^{n}(t)<r+\delta$ for all $n \geq n_{r}$. Putting $s=\phi^{n_{r}}(t)$, we deduce that $\phi^{n_{s}}(s)<r$, i.e., $\phi^{n_{s}+n_{r}}(t)<r$, a contradiction. We conclude that $\lim _{n \rightarrow \infty} \phi^{n}(t)=0$.

Remark 3 Observe that, as a partial converse of the above lemma, if $\phi: \mathbb{R}^{+} \rightarrow \mathbb{R}^{+}$satisfies $\lim _{n \rightarrow \infty} \phi^{n}(t)=0$ for all $t>0$, then $\phi$ is a weaker Meir-Keeler function. Indeed, otherwise, there exist $\varepsilon>0$ and a sequence $\left\{t_{n}\right\}_{n \in \mathbb{N}}$ with $t_{n} \geq \varepsilon$ for all $n \in \mathbb{N}, \lim _{n \rightarrow \infty} t_{n}=\varepsilon$ but $\phi^{k}\left(t_{n}\right) \geq$ $\varepsilon$ for all $k, n \in \mathbb{N}$, a contradiction.

We also will use the following cyclic extension of the celebrated Matkowski fixed point theorem [17, Theorem 1.2], where for a self-map $f$ of a metric space $(X, d)$, we define

$$
M_{d}(x, y)=\max \left\{d(x, y), d(x, f x), d(y, f y), \frac{1}{2}[d(x, f y)+d(f x, y)]\right\}
$$

for all $x, y \in X$.

Theorem 4 ( $c f .[18$, Corollary 2.14]) Let $f$ be a self-map of a complete metric space $(X, d)$, and let $X=\bigcup_{i=1}^{m} A_{i}$ be a cyclic representation of $X$ with respect to $f$, with $A_{i}$ non-empty and closed, $i=1, \ldots$, m. If $\phi: \mathbb{R}^{+} \rightarrow \mathbb{R}^{+}$is a non-decreasing function such that $\lim _{n \rightarrow \infty} \phi^{n}(t)=0$ for all $t>0$, and for any $x \in A_{i}, y \in A_{i+1}, i=1,2, \ldots, m$,

$$
d(f x, f y) \leq \phi\left(M_{d}(x, y)\right)
$$

where $A_{m+1}=A_{1}$, then f has a unique fixed point $z$. Moreover, $z \in \bigcap_{i=1}^{m} A_{i}$.

Then from Lemma 2 and Theorem 4 we immediately deduce the following theorem.

Theorem 5 Let $f$ be a self-map of a complete metric space $(X, d)$, and let $X=\bigcup_{i=1}^{m} A_{i}$ be a cyclic representation of $X$ with respect to $f$, with $A_{i}$ non-empty and closed, $i=1, \ldots, m$. If $\phi: \mathbb{R}^{+} \rightarrow \mathbb{R}^{+}$is a non-decreasing weaker Meir-Keeler function such that for any $x \in A_{i}$, $y \in A_{i+1}, i=1,2, \ldots, m$,

$$
d(f x, f y) \leq \phi\left(M_{d}(x, y)\right)
$$

where $A_{m+1}=A_{1}$, then fhas a unique fixed point $z$. Moreover, $z \in \bigcap_{i=1}^{m} A_{i}$.

Corollary Let $f$ be a self-map of a complete metric space $(X, d)$, and let $X=\bigcup_{i=1}^{m} A_{i}$ be a cyclic representation of $X$ with respect to $f$, with $A_{i}$ non-empty and closed, $i=1, \ldots, m$. 
If $\phi: \mathbb{R}^{+} \rightarrow \mathbb{R}^{+}$is a non-decreasing weaker Meir-Keeler function such that for any $x \in A_{i}$, $y \in A_{i+1}, i=1,2, \ldots, m$,

$$
d(f x, f y) \leq \phi(d(x, y))
$$

where $A_{m+1}=A_{1}$, then f has a unique fixed point $z$. Moreover, $z \in \bigcap_{i=1}^{m} A_{i}$.

Proof Since $\phi$ is non-decreasing, we deduce that for each $x, y \in X, \phi(d(x, y)) \leq \phi\left(M_{d}(x, y)\right)$, so $d(f x, f y) \leq \phi\left(M_{d}(x, y)\right)$. Hence, by Theorem $5, f$ has a unique fixed point $z$ and $z \in$ $\bigcap_{i=1}^{m} A_{i}$.

Theorem 5 can be generalized according to the style of Chen's theorem as follows.

Theorem 6 Let $f$ be a self-map of a complete metric space $(X, d)$, and let $X=\bigcup_{i=1}^{m} A_{i}$ be a cyclic representation of $X$ with respect to $f$, with $A_{i}$ non-empty and closed, $i=1, \ldots, m$. If $\phi: \mathbb{R}^{+} \rightarrow \mathbb{R}^{+}$is a non-decreasing weaker Meir-Keeler function, $\varphi: \mathbb{R}^{+} \rightarrow \mathbb{R}^{+}$is a function satisfying conditions $\left(\varphi_{i}\right), i=1,2,3$, and for any $x \in A_{i}, y \in A_{i+1}, i=1,2, \ldots, m$, it follows

$$
\varphi(d(f x, f y)) \leq \phi\left(\varphi\left(M_{d}(x, y)\right)\right)
$$

where $A_{m+1}=A_{1}$, then f has a unique fixed point $z$. Moreover, $z \in \bigcap_{i=1}^{m} A_{i}$.

Proof Construct the complete metric space $(X, p)$ of Proposition 1 , and observe that from the well-known fact that for $a_{i} \in \mathbb{R}^{+}, i=1, \ldots, k$, one has $\phi\left(\max _{i} a_{i}\right)=\max _{i} \phi\left(a_{i}\right)$, one has

$$
M_{p}(x, y)=\varphi\left(M_{d}(x, y)\right)
$$

for all $x, y \in X$. Therefore, for any $x \in A_{i}, y \in A_{i+1}, i=1,2, \ldots, m$, we deduce that

$$
p(f x, f y) \leq \phi\left(M_{p}(x, y)\right) .
$$

Theorem 5 concludes the proof.

We finish this section with two examples illustrating Theorem 5 and its corollary.

Example 2 Let $A=\{n \in \mathbb{N}: n$ is even $\} \cup\{0\}, B=\{n \in \mathbb{N}: n$ is odd $\} \cup\{0\}, X=A \cup B=\mathbb{N}$, and let $d$ be the complete metric on $X$ defined by $d(x, x)=0$ for all $x \in X$ and $d(x, y)=x+y$ otherwise. Since $d$ induces the discrete topology on $X$, we deduce that $A$ and $B$ are closed subsets of $(X, d)$.

Let $f$ be the self-map of $X$ defined by $f 0=0$ and $f x=x-1$ otherwise. It is clear that $X=A \cup B$ is a cyclic representation of $X$ with respect to $f$.

Now we define the function $\phi: \mathbb{R}^{+} \rightarrow \mathbb{R}^{+}$by $\phi(0)=0$, and $\phi(t)=n-1$ if $t \in(n-1, n]$, $n \in \mathbb{N}$. It is immediate to check that $\phi$ is a non-decreasing weaker Meir-Keeler function which is not a Meir-Keeler function.

Furthermore, we have:

- For $x=0$ and $y=1, d(f x, f y)=d(0,0)=0$. 
- For $x=0$ and $y=n \in \mathbb{N} \backslash\{1\}$,

$$
d(f x, f y)=d(0, n-1)=n-1=\phi(n)=\phi(d(x, y)) .
$$

- For $x=n \in A \backslash\{0\}$ and $y=m \in B \backslash\{0\}$,

$$
\begin{aligned}
d(f x, f y) & =d(n-1, m-1)=n+m-2<n+m-1 \\
& =\phi(n+m)=\phi(d(x, y)) .
\end{aligned}
$$

Consequently, the conditions of the corollary of Theorem 5 are verified; in fact, $z=0 \in$ $A \cap B$ is the unique fixed point of $f$.

Example 3 Let $A=[0,1 / 2] \cup\{1\}, B=[1,2], X=A \cup B$ and let $d$ be the restriction to $X$ of the Euclidean metric on $\mathbb{R}$. Obviously, $(X, d)$ is a complete metric space (in fact, it is compact), with $A$ and $B$ closed subsets of $(X, d)$.

Let $f$ be the self-map of $X$ defined by $f x=2-x$ if $x \in A$, and $f x=1$ if $x \in B$. It is clear that $X=A \cup B$ is a cyclic representation of $X$ with respect to $f$.

Now we define the function $\phi: \mathbb{R}^{+} \rightarrow \mathbb{R}^{+}$by $\phi(t)=t / 2$ if $t \in[0,1]$, and $\phi(t)=1$ if $t>1$. (Notice that $\phi$ is a non-decreasing weaker Meir-Keeler function which is not a Meir-Keeler function.)

Furthermore, we have:

- For $x=1 \in A$ and $y \in B, d(f x, f y)=d(1,1)=0$.

- For $x=1 / 2 \in A$ and $y \in B$,

$$
d(f x, f y)=d(3 / 2,1)=1 / 2=\phi(1)=\phi(d(x, f x)) .
$$

- For $x \in A \backslash\{1,1 / 2\}$ and $y \in B$,

$$
d(f x, f y)=d(2-x, 1)=1-x \leq 1=\phi(2-2 x)=\phi(d(x, f x)) .
$$

Consequently, the conditions of Theorem 5 are verified; in fact, $z=1 \in A \cap B$ is the unique fixed point of $f$.

Finally, observe that the corollary of Theorem 5 cannot be applied in this case because for $x=1 / 2 \in A$ and $y=1 \in B$, we have

$$
d(f x, f y)=1 / 2>\phi(1 / 2)=\phi(d(x, y)) .
$$

\section{Applications to well-posedness and limit shadowing property of a fixed point problem}

The notion of well-posedness of a fixed point problem has evoked much interest to several mathematicians, for example, De Blasi and Myjak [19], Lahiri and Das [20], Popa [21, 22] and others.

Definition 3 [19] Let $f$ be a self-map of a metric space $(X, d)$. The fixed point problem of $f$ is said to be well posed if: 
(i) $f$ has a unique fixed point $z \in X$;

(ii) for any sequence $\left\{x_{n}\right\}_{n \in \mathbb{N}}$ in $X$ such that $\lim _{n \rightarrow \infty} d\left(f x_{n}, x_{n}\right)=0$, we have $\lim _{n \rightarrow \infty} d\left(x_{n}, z\right)=0$.

Definition 4 [22] Let $f$ be a self-map of a metric space $(X, d)$. The fixed point problem of $f$ is said to have limit shadowing property in $X$ if for any sequence $\left\{x_{n}\right\}_{n \in \mathbb{N}}$ in $X$ satisfying $\lim _{n \rightarrow \infty} d\left(f x_{n}, x_{n}\right)=0$, it follows that there exists $z \in X$ such that $\lim _{n \rightarrow \infty} d\left(f^{n} z, x_{n}\right)=0$.

Concerning the well-posedness and limit shadowing of the fixed point problem for a self-map of a complete metric space satisfying the conditions of Theorem 5 , we have the following results.

Theorem 7 Let $(X, d)$ be a complete metric space. Iff is a self-map of $X$ and $\phi: \mathbb{R}^{+} \rightarrow \mathbb{R}^{+}$is a non-decreasing weaker Meir-Keeler function satisfying the conditions of Theorem 5, then the fixed point problem of $f$ is well posed.

Proof Owing to Theorem 5, we know that $f$ has a unique fixed point $z \in X$. Let $\left\{x_{n}\right\}$ be a sequence in $X$ such that $\lim _{n \rightarrow \infty} d\left(x_{n}, f x_{n}\right)=0$. Then

$$
\begin{aligned}
d\left(x_{n}, z\right) \leq & d\left(x_{n}, f x_{n}\right)+d\left(f x_{n}, f z\right) \\
\leq & d\left(x_{n}, f x_{n}\right) \\
& +\phi\left(\max \left\{d\left(x_{n}, z\right), d\left(x_{n}, x_{n+1}\right), d(z, f z), \frac{d\left(x_{n}, f z\right)+d\left(z, x_{n+1}\right)}{2}\right\}\right) .
\end{aligned}
$$

Passing to the limit as $n \rightarrow \infty$ in the above inequality, it follows that $\lim _{n \rightarrow \infty} d\left(x_{n}, z\right)=0$.

Theorem 8 Let $(X, d)$ be a complete metric space. Iff is a self-map of $X$ and $\phi: \mathbb{R}^{+} \rightarrow \mathbb{R}^{+}$is a non-decreasing weaker Meir-Keeler function satisfying the conditions of Theorem 5, then $f$ has the limit shadowing property.

Proof From Theorem 5, we know that $f$ has a unique fixed point $z \in X$. Let $\left\{x_{n}\right\}_{n \in \mathbb{N}}$ be a sequence in $X$ such that $\lim _{n \rightarrow \infty} d\left(x_{n}, f x_{n}\right)=0$. Then, as in the proof of the previous theorem,

$$
\begin{aligned}
d\left(x_{n}, z\right) \leq & d\left(x_{n}, f x_{n}\right) \\
& +\phi\left(\max \left\{d\left(x_{n}, z\right), d\left(x_{n}, x_{n+1}\right), d(z, f z), \frac{d\left(x_{n}, f z\right)+d\left(z, x_{n+1}\right)}{2}\right\}\right) .
\end{aligned}
$$

Passing to the limit as $n \rightarrow \infty$ in the above inequality, it follows that $\lim _{n \rightarrow \infty} d\left(x_{n}, f^{n} z\right)=$ $d\left(x_{n}, z\right)=0$.

\section{An application to integral equations}

In this section we apply Theorem 5 to study the existence and uniqueness of solutions for a class of nonlinear integral equations. 
We consider the nonlinear integral equation

$$
u(t)=\int_{0}^{T} G(t, s) K(s, u(s)) d s \quad \text { for all } t \in[0, T]
$$

where $T>0, K:[0, T] \times \mathbb{R}^{+} \rightarrow \mathbb{R}^{+}$and $G:[0, T] \times[0, T] \rightarrow \mathbb{R}^{+}$are continuous functions, and $M:=\max _{(s, x) \in[0, T]^{2}} K(s, x)$.

We shall suppose that the following four conditions are satisfied:

(I) $\int_{0}^{T} G(t, s) d s \leq 1$ for all $t \in[0, T]$.

(II) $K(s, \cdot)$ is a non-increasing function for any fixed $s \in[0,1]$, that is,

$$
x, y \in \mathbb{R}^{+}, \quad x \geq y \quad \Longrightarrow \quad K(s, x) \leq K(s, y) .
$$

(III) There exists a Meir-Keeler function $\psi: \mathbb{R}^{+} \rightarrow \mathbb{R}^{+}$that is non-decreasing on $[0,2 M]$ and such that

$$
|K(s, x)-K(s, y)| \leq \psi(|x-y|)
$$

for all $s, x \in[0, T]$ and $y \in \mathbb{R}^{+}$with $|x-y| \leq 2 M$.

(IV) There exists a continuous function $\alpha:[0, T] \rightarrow[0, T]$ such that:

For all $t \in[0, T]$, we have

$$
\alpha(t) \leq \int_{0}^{T} G(t, s) K(s, T) d s
$$

and

$$
T \geq \int_{0}^{T} G(t, s) K(s, \alpha(s)) d s .
$$

Now denote by $C\left([0, T], \mathbb{R}^{+}\right)$the set of non-negative real continuous functions on $[0, T]$. We endow $C\left([0, T], \mathbb{R}^{+}\right)$with the supremum metric

$$
d_{\infty}(u, v)=\max _{t \in[0, T]}|u(t)-v(t)|, \quad \text { for all } u, v \in C\left([0, T], \mathbb{R}^{+}\right) .
$$

It is well known that $\left(C\left([0, T], \mathbb{R}^{+}\right), d_{\infty}\right)$ is a complete metric space.

Consider the self-map $f: C\left([0, T], \mathbb{R}^{+}\right) \rightarrow C\left([0, T], \mathbb{R}^{+}\right)$defined by

$$
f u(t)=\int_{0}^{T} G(t, s) K(s, u(s)) d s \quad \text { for all } t \in[0, T] .
$$

Clearly, $u$ is a solution of (1) if and only if $u$ is a fixed point of $f$.

In order to prove the existence of a (unique) fixed point of $f$, we construct the closed subsets $A_{1}$ and $A_{2}$ of $C\left([0, T], \mathbb{R}^{+}\right)$as follows:

$$
A_{1}=\left\{u \in C\left([0, T], \mathbb{R}^{+}\right): u(s) \leq T \text { for all } s \in[0, T]\right\},
$$

and

$$
A_{2}=\left\{u \in C\left([0, T], \mathbb{R}^{+}\right): u \geq \alpha\right\} .
$$


We shall prove that

$$
f\left(A_{1}\right) \subseteq A_{2} \quad \text { and } \quad f\left(A_{2}\right) \subseteq A_{1} .
$$

Let $u \in A_{1}$, that is,

$$
u(s) \leq T \quad \text { for all } s \in[0, T] .
$$

Since $G(t, s) \geq 0$ for all $t, s \in[0, T]$, we deduce from (II) and (IV) that

$$
\int_{0}^{T} G(t, s) K(s, u(s)) d s \geq \int_{0}^{T} G(t, s) K(s, T) d s \geq \alpha(t)
$$

for all $t \in[0, T]$. Then we have $f u \in A_{2}$.

Similarly, let $u \in A_{2}$, that is,

$$
u(s) \geq \alpha(s) \quad \text { for all } s \in[0, T]
$$

Again, from (II) and (IV), we deduce that

$$
\int_{0}^{T} G(t, s) K(s, u(s)) d s \leq \int_{0}^{T} G(t, s) K(s, \alpha(s)) d s \leq T
$$

for all $t \in[0, T]$. Then we have $f u \in A_{1}$. Thus, we have shown that (2) holds.

Hence, if $X:=A_{1} \cup A_{2}$, we have that $X$ is closed in $C\left([0, T], \mathbb{R}^{+}\right)$, so the metric space $\left(X, d_{\infty}\right)$ is complete.

Moreover, $X:=A_{1} \cup A_{2}$ is a cyclic representation of the restriction of $f$ with respect to $X$, which will be also denoted by $f$.

Now construct the function $\phi: \mathbb{R}^{+} \rightarrow \mathbb{R}^{+}$given by

$$
\phi(t)=\psi(t) \quad \text { if } t \in[0,2 M],
$$

and

$$
\phi(t)=2 M \quad \text { if } t>2 M .
$$

Since $\psi$ is a Meir-Keeler function that is non-decreasing on $[0,2 M]$, it immediately follows that $\phi$ is a non-decreasing weaker Meir-Keeler function. Note also that $\phi$ is not continuous at $t=2 M$ (in fact, it is not a Meir-Keeler function).

Finally we shall show that for each $u \in A_{1}$ and $v \in A_{2}$, one has $d_{\infty}(f u, f v) \leq \phi\left(d_{\infty}(u, v)\right)$.

To this end, let $u \in A_{1}$ and $v \in A_{2}$. Since $u(s) \in[0, T]$ for each $s \in[0, T]$, we have that

$$
\begin{aligned}
f u(t) & =\int_{0}^{T} G(t, s) K(s, u(s)) d s \\
& \leq M \int_{0}^{T} G(t, s) d s \leq M
\end{aligned}
$$

for all $t \in[0, T]$. 
Similarly, since $v \geq \alpha$ and $\alpha(s) \in[0, T]$ for each $s \in[0, T]$, we deduce that

$$
f v(t) \leq \int_{0}^{T} G(t, s) K(s, \alpha(s)) d s \leq M
$$

for all $t \in[0, T]$. Therefore

$$
\mid f u(t)-f(v(t) \mid \leq f u(t)+f v(t) \leq 2 M
$$

for all $t \in[0, T]$. So,

$$
d_{\infty}(f u, f v) \leq 2 M
$$

If $d_{\infty}(u, v)>2 M$, we have $\phi\left(d_{\infty}(u, v)\right)=2 M$, so

$$
d_{\infty}(f u, f v) \leq \phi\left(d_{\infty}(u, v)\right) .
$$

If $d_{\infty}(u, v) \leq 2 M$, then $|u(s)-v(s)| \leq 2 M$ for all $s \in[0, T]$, so by (III), we deduce that for each $t \in[0, T]$,

$$
\begin{aligned}
\mid f u(t)-f(v(t) \mid & \leq \int_{0}^{T} G(t, s)|K(s, u(s))-K(s, v(s))| d s \\
& \leq \int_{0}^{T} G(t, s) \psi(|u(s)-v(s)|) d s \\
& \leq \psi\left(d_{\infty}(u, v)\right) \int_{0}^{T} G(t, s) d s \\
& \leq \psi\left(d_{\infty}(u, v)\right) \\
& =\phi\left(d_{\infty}(u, v)\right) .
\end{aligned}
$$

Consequently, by the corollary of Theorem $5, f$ has a unique fixed point $u^{*} \in A_{1} \cap A_{2}$, that is, $u^{*} \in \mathcal{C}$ is the unique solution to (1) in $A_{1} \cup A_{2}$.

Remark 4 The first author studied in [9, Section 3] a variant of the problem discussed above for the case that $\psi$ is the non-decreasing Meir-Keeler function given by $\psi(t)=$ $\left(\ln \left(t^{2}+1\right)\right)^{1 / 2}$ for all $t \in \mathbb{R}^{+}$.

The next example illustrates the preceding development.

Example 4 Consider the integral equation

$$
u(t)=\int_{0}^{T} G(t, s) K(s, u(s)) d s \quad \text { for all } t \in[0, T]
$$

where $T=1, G(t, s)=t$ for all $t, s \in[0,1]$, and

$$
K(s, x)=\frac{\cos s}{1+x}
$$

for all $s \in[0,1]$ and $x \geq 0$. 
Hence, $M=\max _{(s, x) \in[0,1]^{2}} K(s, x)=K(0,0)=1$.

Furthermore, it is obvious that $G$ satisfies condition (I), whereas $K$ satisfies condition (II).

Now construct a Meir-Keeler function $\psi: \mathbb{R}^{+} \rightarrow \mathbb{R}^{+}$as

$$
\psi(t)=t /(1+t) \quad \text { if } t \in[0,2]
$$

and

$$
\psi(t)=0 \quad \text { if } t>2
$$

Note that $\psi$ is non-decreasing on $[0,2]$ and not continuous at $t=2$.

Moreover, for each $s, x \in[0,1]$ and each $y \in \mathbb{R}^{+}$with $|x-y| \leq 2$, we have

$$
|K(s, x)-K(s, y)|=\cos s\left|\frac{1}{1+x}-\frac{1}{1+y}\right| \leq \frac{|x-y|}{1+|x-y|}=\psi(|x-y|)
$$

so condition (III) is also satisfied.

Finally, define $\alpha:[0,1] \rightarrow[0,1]$ as $\alpha(t)=t / 3$ for all $t \in[0,1]$. It is not hard to check that $\alpha$ verifies condition (IV), and consequently the integral equation has a unique solution $u^{*}$ in $A_{1} \cup A_{2}$, where $A_{1}=\left\{u \in C\left([0,1], \mathbb{R}^{+}\right): u(s) \leq 1\right.$ for all $\left.s \in[0,1]\right\}$ and $A_{2}=\left\{u \in C\left([0,1], \mathbb{R}^{+}\right)\right.$: $u(s) \geq s / 3$ for all $s \in[0,1]\}$. In fact $u^{*} \in A_{1} \cap A_{2}$, i.e., $t / 3 \leq u^{*}(t) \leq 1$ for all $t \in[0,1]$.

Note that, according to our constructions, for each pair $u, v \in C\left([0,1], \mathbb{R}^{+}\right)$with $u \leq 1$ and $v \geq \alpha$, we have $d_{\infty}(f u, f v) \leq \phi\left(d_{\infty}(u, v)\right)$, where $\phi$ is the non-decreasing weaker MeirKeeler function defined as $\phi(t)=t /(t+1)$ if $t \in[0,2]$ and $\phi(t)=2$ if $t>2$.

In particular, we can deduce the following approximation to the value of $u^{*}(t)$ for each $t \in[0,1]$ :

$$
\begin{aligned}
\left|u^{*}(t)-\frac{\sin 1}{2} t\right| & =\left|u^{*}(t)-\int_{0}^{1} t \frac{\cos s}{2} d s\right|=\left|f u^{*}(t)-\int_{0}^{1} G(t, s) K(s, 1) d s\right| \\
& \leq \phi\left(d_{\infty}\left(u^{*}, 1\right)\right)=\frac{\max _{t \in[0,1]}\left(1-u^{*}(t)\right)}{1+\max _{t \in[0,1]}\left(1-u^{*}(t)\right)} \\
& =\frac{1-\min _{t \in[0,1]} u^{*}(t)}{2-\min _{t \in[0,1]} u^{*}(t)} \\
& \leq \frac{1}{2}
\end{aligned}
$$

Note also that the contraction inequality $d_{\infty}(f u, f v) \leq \phi\left(d_{\infty}(u, v)\right)$ does not follow when the weaker Meir-Keeler function $\phi$ is replaced by our initial Meir-Keeler function $\psi$ : Take, for instance, the constant functions $u=0$ and $v=3$; then $u \leq 1, v \geq \alpha$, and

$$
\psi\left(d_{\infty}(u, v)\right)=\psi(3)=0<d_{\infty}(f u, f v) .
$$

Remark 5 In Example 4 above, the inequality $|K(s, x)-K(s, y)| \leq \psi(|x-y|)$ is not globally satisfied, i.e., there exist $s, x \in[0,1]$ and $y \in \mathbb{R}^{+}$such that $|K(s, x)-K(s, y)|>\psi(|x-y|)$. In fact, this happens for all $x, y \in \mathbb{R}^{+}$with $y>x+2$. However, it is clear that for each $s \in[0,1]$, and $x, y \in \mathbb{R}^{+}$, one has $|K(s, x)-K(s, y)| \leq \psi_{1}(|x-y|)$ for all $s \in[0,1]$, and $x, y \in \mathbb{R}^{+}$, where $\psi_{1}(t)=t /(t+1)$ for all $t \in \mathbb{R}^{+}$. 
We conclude the paper with an example where conditions (I)-(IV) also hold (in particular, (III) for the function $\psi_{1}$ defined above) but the inequality $|K(s, x)-K(s, y)| \leq \psi_{1}(|x-y|)$ is not globally satisfied.

Example 5 We modify Example 4 as follows. Consider the integral equation

$$
u(t)=\int_{0}^{T} G(t, s) K(s, u(s)) d s \quad \text { for all } t \in[0, T]
$$

where $T=2, G(t, s)=t / 2$ for all $t, s \in[0,2]$, and

$$
\begin{aligned}
& K(s, x)=e^{-s} /(1+x) \quad \text { if } s \in[0,2], x \in[0,1] ; \\
& K(s, x)=e^{-s} /\left(1+x^{1 / 2}\right) \quad \text { if } s \in[0,2], x \in(1,4] ; \\
& K(s, x)=e^{-s} /(4 x-13) \quad \text { if } s \in[0,2], x>4 .
\end{aligned}
$$

Clearly $K$ is continuous on $[0,2] \times \mathbb{R}^{+}$. Moreover, $M=1$, and $G$ and $K$ satisfy conditions (I) and (II), respectively.

Now, construct a Meir-Keeler function $\psi_{1}: \mathbb{R}^{+} \rightarrow \mathbb{R}^{+}$as $\psi_{1}(t)=t /(1+t)$ for all $t \in \mathbb{R}^{+}$. By discussing the different cases, it is routine to show that for each $s, x \in[0,2]$ and each $y \in \mathbb{R}^{+}$with $|x-y| \leq 2$, we have

$$
|K(s, x)-K(s, y)| \leq \psi_{1}(|x-y|),
$$

so condition (III) is also satisfied.

Finally, define $\alpha:[0,2] \rightarrow[0,2]$ as $\alpha(t)=6 t / 35$ for all $t \in[0,2]$. Then, for each $t \in[0,2]$, we have

$$
\int_{0}^{2} G(t, s) K(s, 2) d s=\frac{t}{2} \int_{0}^{2} \frac{e^{-s}}{1+\sqrt{2}} d s=t \frac{\left(1-e^{-2}\right)}{2(1+\sqrt{2})}>\frac{6 t / 7}{5}=\alpha(t) .
$$

Now observe that $\alpha(s)<1$ for all $s \in[0,2]$, so $K(s, \alpha(s))=e^{-s} /(1+\alpha(s))$. Hence, for each $t \in[0,2]$,

$$
\begin{aligned}
\int_{0}^{2} G(t, s) K(s, \alpha(s)) d s & =\frac{t}{2} \int_{0}^{2} \frac{e^{-s}}{1+(6 s / 35)} d s=\frac{t}{2} \int_{0}^{2} \frac{35 e^{-s}}{35+6 s} d s \\
& \leq \frac{t}{2} \int_{0}^{2} d s=t \leq 2 .
\end{aligned}
$$

Therefore $\alpha$ verifies condition (IV), and consequently the integral equation has a unique solution $u^{*}$ in $A_{1} \cup A_{2}$, where $A_{1}=\left\{u \in C\left([0,1], \mathbb{R}^{+}\right): u(s) \leq 2\right.$ for all $\left.s \in[0,2]\right\}$ and $A_{2}=$ $\left\{u \in C\left([0,1], \mathbb{R}^{+}\right): u(s) \geq 6 s / 35\right.$ for all $\left.s \in[0,2]\right\}$. In fact $u^{*} \in A_{1} \cap A_{2}$, i.e., $6 t / 35 \leq u^{*}(t) \leq 2$ for all $t \in[0,2]$.

It is interesting to observe that the Meir-Keeler function $\psi_{1}$ is continuous on $\mathbb{R}^{+}$but condition (III) is not globally satisfied: Indeed, take $x=0$ and $y>14 / 3$. Then, for each 
$s \in[0,1]$, we obtain

$$
K(s, x)-K(s, y)=e^{-s}\left(1-\frac{1}{4 y-13}\right)>e^{-s} \frac{y}{1+y} .
$$

Hence, $K(0,0)-K(0, y)>\psi_{1}(y)$.

\section{Competing interests}

The authors declare that they have no competing interests.

\section{Authors' contributions}

The two authors contributed equally in writing this article. They read and approved the final manuscript.

\section{Author details}

${ }^{1}$ Department of Mathematics, Disha Institute of Management and Technology, Satya Vihar, Vidhansabha-Chandrakhuri Marg, Mandir Hasaud, Raipur, Chhattisgarh 492101, India. ${ }^{2}$ Instituto Universitario de Matemática Pura y Aplicada, Universitat Politècnica de València, Camí de Vera s/n, Valencia, 46022, Spain.

\section{Acknowledgements}

The second author thanks for the support of the Ministry of Economy and Competitiveness of Spain under grant MTM2012-37894-C02-01, and the Universitat Politècnica de València, grant PAID-06-12-SP20120471.

Received: 7 May 2013 Accepted: 5 August 2013 Published: 22 August 2013

\section{References}

1. Kirk, WA, Srinavasan, PS, Veeramani, P: Fixed points for mapping satisfying cyclical contractive conditions. Fixed Point Theory 4, 79-89 (2003)

2. Banach, S: Sur les operations dans les ensembles abstraits et leur application aux equations integerales. Fundam. Math. 3, 133-181 (1922)

3. Boyd, DW, Wong, SW: On nonlinear contractions. Proc. Am. Math. Soc. 20, 458-464 (1969)

4. Caristi, J: Fixed point theorems for mappings satisfying inwardness conditions. Trans. Am. Math. Soc. 215, 241-251 (1976)

5. Di Bari, C, Suzuki, T, Vetro, C: Best proximity points for cyclic Meir-Keeler contractions. Nonlinear Anal. 69, 3790-3794 (2008)

6. Karapinar, E: Fixed point theory for cyclic weaker $\phi$-contraction. Appl. Math. Lett. 24, 822-825 (2011)

7. Karapinar, E, Sadarangani, K: Corrigendum to "Fixed point theory for cyclic weaker $\phi$-contraction" [Appl. Math. Lett. Vol. 24(6), 822-825.]. Appl. Math. Lett. 25, 1582-1584 (2012)

8. Karapinar, E, Sadarangani, K: Fixed point theory for cyclic $(\phi-\varphi)$-contractions. Fixed Point Theory Appl. 2011, 69 (2011)

9. Nahsine, HK: Cyclic generalized $\psi$-weakly contractive mappings and fixed point results with applications to integral equations. Nonlinear Anal. 75, 6160-6169 (2012)

10. Păcurar, M: Fixed point theory for cyclic Berinde operators. Fixed Point Theory 12,419-428 (2011)

11. Păcurar, M, Rus, IA: Fixed point theory for cyclic $\varphi$-contractions. Nonlinear Anal. 72, 2683-2693 (2010)

12. Piatek, B: On cyclic Meir-Keeler contractions in metric spaces. Nonlinear Anal. 74, 35-40 (2011)

13. Rus, IA: Cyclic representations and fixed points. Ann. "Tiberiu Popoviciu” Sem. Funct. Equ. Approx. Convexity 3, 171-178 (2005)

14. Chen, CM: Fixed point theory for the cyclic weaker Meir-Keeler function in complete metric spaces. Fixed Point Theory Appl. 2012, 17 (2012)

15. Chen, CM: Fixed point theorems for cyclic Meir-Keeler type mappings in complete metric spaces. Fixed Point Theory Appl. 2012, 41 (2012)

16. Meir, A, Keeler, E: A theorem on contraction mappings. J. Math. Anal. Appl. 28, 326-329 (1969)

17. Matkowski, J: Integrable solutions of functional equations. Diss. Math. 127, 1-68 (1975)

18. Karapinar, E, Romaguera, S, Tas, K: Fixed points for cyclic orbital generalized contractions on complete metric spaces. Cent. Eur. J. Math. 11, 552-560 (2013)

19. De Blasi, FS, Myjak, J: Sur la porosité des contractions sans point fixed. C. R. Math. Acad. Sci. Paris 308, 51-54 (1989)

20. Lahiri, BK, Das, P: Well-posedness and porosity of certain classes of operators. Demonstr. Math. 38, 170-176 (2005)

21. Popa, V: Well-posedness of fixed point problems in orbitally complete metric spaces. Stud. Cercet. ştiinţ. - Univ. Bacău Ser. Mat. Supplement. Proceedings of ICMI 45, Bacau, Sept. 18-20 (2006) 16, 209-214 (2006)

22. Popa, W: Well-posedness of fixed point problems in compact metric spaces. Bul. Univ. Petrol-Gaze, Ploiesti, Sec. Mat. Inform. Fiz. 60, 1-4 (2008)

doi:10.1186/1687-1812-2013-224

Cite this article as: Nashine and Romaguera: Fixed point theorems for cyclic self-maps involving weaker Meir-Keeler functions in complete metric spaces and applications. Fixed Point Theory and Applications 2013 2013:224. 